\title{
Incidencia de la actitud conductual en las intenciones emprendedoras en estudiantes universitarios
}

\author{
Incidence of the behavioral attitude in the entrepreneurial \\ intentions in undergraduate students
}

\begin{abstract}
Said Diez Farhat*, Rubén Guevara
CENTRUM Católica Graduate Business School, Pontificia Universidad Católica del Perú, Perú
\end{abstract}

Recibido el 22 de junio de 2018; aceptado el 21 de enero de 2019

Disponible en Internet el: 24 de enero de 2019

\section{Resumen}

Las intenciones emprendedoras han sido un tema estudiado con frecuencia por la comunidad científica. La literatura existente expresa argumentos variados, en el cual el tema central es como se determina la intención emprendedora. El presente estudio incluyó tres propósitos específicos. El primero fue explicar la incidencia de la proactividad en las intenciones emprendedoras en los estudiantes de pregrado; el segundo fue explicar la incidencia de la propensión al riesgo en las intenciones emprendedoras en los estudiantes de pregrado y el tercero fue explicar la incidencia de la autoeficacia en las intenciones emprendedoras en los estudiantes de pregrado. La recolección de datos fue realizada en ocho universidades ubicadas en las tres provincias de mayor población, importancia económica y número de estudiantes universitarios en el Ecuador. Mediante el análisis usando modelos de ecuaciones estructurales se demostró que la autoeficacia como factor reflectivo de segundo orden y la proactividad como factor reflectivo unidimensional, tienen una influencia significativa en la intención emprendedora de los estudiantes universitarios de Ecuador.

\footnotetext{
*Autor para correspondencia

Correo electrónico said.diez@cu.ucsg.edu.ec (S. Diez Farhat).

La revisión por pares es responsabilidad de la Universidad Nacional Autónoma de México.

http://dx.doi.org/10.22201/fca.24488410e.2019.2074

0186- 1042/@ 2019 Universidad Nacional Autónoma de México, Facultad de Contaduría y Administración. Este es un artículo Open Access bajo la licencia CC BY-NC-SA (https://creativecommons.org/licenses/by-nc-sa/4.0/)
} 
Palabras clave: Proactividad; Autoeficacia; Propensión al riesgo; Emprendimiento; Intención emprendedora

\begin{abstract}
Entrepreneurial intentions have been a subject frequently studied by the scientific community. Entrepreneurial intentions have been a subject frequently studied by the scientific community. The existing literature expresses varied arguments, in which the central theme is how entrepreneurial intention is determined. The study presented three specific purposes. The first was to explain the incidence of attitudes toward behavior in entrepreneurial intentions in undergraduate students; the second was to explain the incidence of subjective norms on entrepreneurial intentions in undergraduate students and the third was to explain the incidence of perceived control in entrepreneurial intentions in undergraduate students. The data collection was carried out in 8 universities located in the three provinces with the largest population, economic importance and numbers of university students in Ecuador. Through the analysis using structural equation models, it was demonstrated that self-efficacy as a second-order reflective factor and proactivity as a one-dimensional reflective factor, have a significant influence on the entrepreneurial intention of university students in Ecuador.
\end{abstract}

JEL code: M10, M13, M16

Keywords: Proactivity; Self-efficacy; Risk-proneness; Entrepreneurship; Entrepreneurial intention

\title{
Introducción
}

Existe una colectividad considerable de la literatura que aborda el concepto de las intenciones empresariales desde finales de 1980, viendo gran parte de la actividad empresarial, como la conducta intencional y la formación de una intención de iniciar un negocio como un paso en el proceso de creación de una organización (Bird, 1988; Krueger, Reilly, \& Carsrud, 2000; Van Gelderen et al., 2008). El marco teórico utilizado comúnmente en esta corriente de investigación (Schlaegel \& Koenig, 2012; 2014) es la teoría del comportamiento planificado (TPB), que conceptualiza la fuerza de la intención como un antecedente inmediato del comportamiento (Ajzen, 1991; 2011). En los siguientes años, se han desarrollado algunos modelos, entre los cuales están el Modelo del Evento Emprendedor (Shapero \& Sokol, 1982), el Modelo de la Orientación Emprendedora (Covin \& Slevin, 1989) y el Modelo del Potencial Emprendedor (Krueger \& Brazeal, 1994).

Algunos autores han estudiado el impacto y el rol del emprendedor en el desarrollo de las sociedades desde el siglo XII y por su importancia se han investigado sus características 
y antecedentes. Por otro lado, la conducta emprendedora es una línea de investigación que debe ser considerada para analizar la influencia de las variables que puedan incidir en las intenciones emprendedoras en los estudiantes universitarios. Sin embargo, en la actualidad no existe un consenso sobre los factores que inciden en la creación de un emprendimiento, lo que da la pauta para establecer los lineamientos académicos para disminuir la escasez de este tipo actividad empresarial (Diez, 2016).

A nivel mundial el desarrollo académico y su evolución a través de la formación de empresarios en los estudiantes universitarios de los diferentes campos de educación, se ha detectado el bajo nivel en la incidencia de las actitudes conductuales que generen intenciones emprendedoras (Arasteh, Enayati, Zameni, \& Khademloo, 2012; Verheul et al., 2015; Zhang, Wang, \& Owen, 2015). Debido a que es muy complejo medir los niveles motivacionales de las "actitudes conductuales" en las intenciones emprendedoras, además de la percepción de problemas en el desarrollo de las mismas, es imperioso dejar un precedente académico de las pautas aplicables en las intenciones emprendedoras en los estudiantes universitarios (Al Mamun, Binti Che Nawi, Dewiendren, \& Fazira Binti, 2016; Frese \& Gielnik, 2014; Lanero, Vázquez, \& Muñoz-Adánez, 2015; Shirokova, Osiyevskyy, \& Bogatyreva, 2015; Soria-Barreto, Zuniga-Jara, \& Ruiz-Campo, 2016; Zhang et al., 2015)Brown and Hackett (1994, 2000. Este estudio se planteó y ejecutó a partir de la Teoría del Comportamiento Planificado de Ajzen (1991), desarrollada específicamente para las investigaciones sobre las intenciones. Por esta razón, esta investigación explicó la incidencia de la actitud hacia el comportamiento, la norma subjetiva y el control de comportamiento percibido en las intenciones emprendedoras, focalizándose en los estudiantes universitarios de pregrado, con el fin de expandir el conocimiento existente en torno a este asunto y, por derivación, proveer de conocimiento comprobado a los futuros emprendedores y al gobierno, en torno a los emprendedores jóvenes y sobre sus iniciativas de generación de riqueza y bienestar mediante los nuevos emprendimientos. Para esta investigación se recopiló información sobre las actitudes conductuales de los estudiantes universitarios de pregrado, mediante un muestreo no probabilístico voluntario, para determinar la existencia de intenciones emprendedoras en jóvenes universitarios.

Esta investigación utilizó técnicas del análisis multivariante de interrelaciones causales entre variables observables y no observables. La investigación se focalizó en tres propósitos específicos. El primero fue explicar la incidencia de la proactividad en las intenciones emprendedoras en los estudiantes de pregrado; el segundo fue explicar la incidencia de la propensión al riesgo en las intenciones emprendedoras en los estudiantes de pregrado; y el tercero fue explicar la incidencia de la autoeficacia en las intenciones emprendedoras en los 
estudiantes de pregrado. Como resultado de los propósitos específicos arriba descritos, se logró determinar la existencia de intenciones emprendedoras, generando un insumo para la academia, los investigadores y los formuladores de políticas públicas relacionadas con nuevos emprendimientos.

\section{Marco teórico}

Desde finales de 1980, la literatura ha abordado el concepto de las intenciones empresariales, viendo gran parte de la actividad empresarial como la conducta intencional y la formación de una intención de iniciar un negocio como un paso en el proceso en la creación y lanzamiento de un nuevo emprendimiento (Bird, 1988; Krueger, Reilly, \& Carsrud, 2000; Van Gelderen et al, 2008). El marco teórico más utilizado desde inicios de los años 1990 en esta corriente de investigación (Schlaegel \& Koenig, 2012; 2014) es la Teoría del Comportamiento Planificado (TPB), que conceptualiza la fuerza de la intención como un antecedente inmediato del comportamiento (Ajzen, 1991; 2011). Esta teoría estipula que las intenciones que preceden a cualquier tipo de comportamiento planificado se determinan por tres factores: (a) la actitud hacia el comportamiento, (b) la norma subjetiva y (c) el control de comportamiento percibido. Es importante destacar que la TPB (Ajzen, 1991), es una extensión de la Teoría de Acción Razonada (Ajzen \& Fishbein, 1980; Fishbein \& Ajzen, 1975), la cual fue modificada por su creador debido a las limitaciones que presentaba el modelo original en la interpretación de los comportamientos de los individuos (Ajzen, 1991, 2011; Ajzen \& Fishbein, 1980; Fishbein \& Ajzen, 1975).

Al igual que en la Teoría original de la Acción Razonada, un factor central en la TPB es la intención del individuo para realizar un determinado comportamiento (Ajzen, 1991, 2011; Ajzen \& Fishbein, 1980; Fishbein \& Ajzen, 1975). Las intenciones en ambas teorías son asumidas para capturar los factores motivacionales que influyen en un comportamiento; son indicaciones de cómo las personas están dispuestas a tratar, de la cantidad de esfuerzo que están planeando para ejercer y con el fin de realizar la conducta. Ambas teorías indican que una intención de conducta puede encontrar expresión en el comportamiento sólo si el comportamiento en cuestión está bajo un control deseado (Ajzen, 1991, 2011; Ajzen \& Fishbein, 1980; Fishbein \& Ajzen, 1975).

De acuerdo a Ajzen (1991), la TPB proporciona un marco conceptual útil para hacer frente a las complejidades de la conducta social humana. Este autor indicó que la teoría incorpora algunos de los conceptos de las ciencias sociales y de comportamiento, y que define estos conceptos de una manera que permite predecir y entender las conductas particulares en contextos 
especificados. Agregó que las actitudes hacia el comportamiento, las normas subjetivas con respecto al comportamiento, y percepción de control sobre el comportamiento son algunas de las variables que se encuentran para predecir las intenciones de comportamiento con un alto grado de precisión.

En esta investigación se refiere a la intención de un comportamiento, por lo cual se debe definir con precisión el criterio de comportamiento. De acuerdo a Ajzen y Fishbein (1980) las actitudes generales no permiten predecir comportamientos específicos por una falta de compatibilidad en los elementos de: (a) acción, (b) contexto y (c) tiempo. Es decir, las actitudes generales identifican sólo el elemento objetivo, mientras que un comportamiento específico implica una acción particular dirigida en el objetivo en un contexto dado y en un punto en el tiempo. La falta de compatibilidad no suele ser un problema serio cuando se trata de predecir el comportamiento de las intenciones porque las medidas de intención no se dirigen a un objetivo general sino a un comportamiento de interés (Israr \& Hashim, 2015; Schlaegel \& Koenig 2014). De hecho, en la literatura existente, los meta-análisis de la relación intención-conducta han revelado generalmente altas correlaciones y que queda explícito la aplicación de la acción, contexto y tiempo (Israr \& Hashim, 2015; Liñán \& Fayolle, 2015; Schlaegel \& Koenig, 2014). Es por ello que la TPB se ha convertido en uno de los modelos predictivos de mayor influencia en la literatura existente (Ajzen, 2011). Los tres factores en los que descansa esta teoría son:

- Creencias conductuales: representa la evaluación personal de aceptación o rechazo a realizar una determinada acción, a este factor se le denomina actitud hacia el comportamiento.

- Creencias normativas: representa el reflejo de la influencia externa y se expresa por medio de la percepción que tiene el individuo acerca de los factores exógenos para realizar o no un comportamiento, también incluye las creencias de aceptación o rechazo de referentes individuales o grupales hacia un determinado comportamiento, a este factor se le denomina normas subjetivas.

- Creencias de control: representa las experiencias pasadas en relación al comportamiento, en este factor también se incluye toda la información que el individuo posee previo a realizar la acción, a este factor se le denomina control conductual percibido

De acuerdo con la TPB (Ajzen, 1991, 2011), los tres factores interactúan entre sí para conformar la intención a realizar una acción. Además, señala que los individuos procesan la información disponible conceptualizada por variables de fondo, de tal forma que la intención para realizar una acción se puede pronosticar a partir de la relación entre las actitudes hacia el comportamiento, las normas subjetivas y el control conductual percibido. Por último, la 
mayoría de modelos sobre intenciones emprendedoras toman como referencia la TPB de Ajzen (1991). En esta línea, varios investigadores han desarrollado modelos de intenciones en el ámbito emprendedor (Al Mamun et al., 2016; Ambad \& Damit, 2016; Fayolle \& Liñán, 2014; Ferreira et al., 2012; Hussain \& Hashim, 2015; Mustafa et al., 2016; Zhang et al., 2015). Existen modelos que indican que la decisión de iniciar una actividad emprendedora requiere de una creencia preexistente de que dicha actividad es deseable y factible (autoeficacia), unida con alguna propensión personal a actuar sobre oportunidades (proactividad) y algún tipo de factor precipitante (propensión al riesgo) (Kakouris, 2016; Küttim, Kallaste, Venesaar, \& Kiis, 2014; Liñán \& Fayolle, 2015; Sánchez, 2011; Sánchez, Lanero, \& Yurrebaso, 2005; Schlaegel \& Koenig, 2014).

Tomando en cuenta esos resultados, la Figura 1 detalla el modelo que se planteó en esta investigación

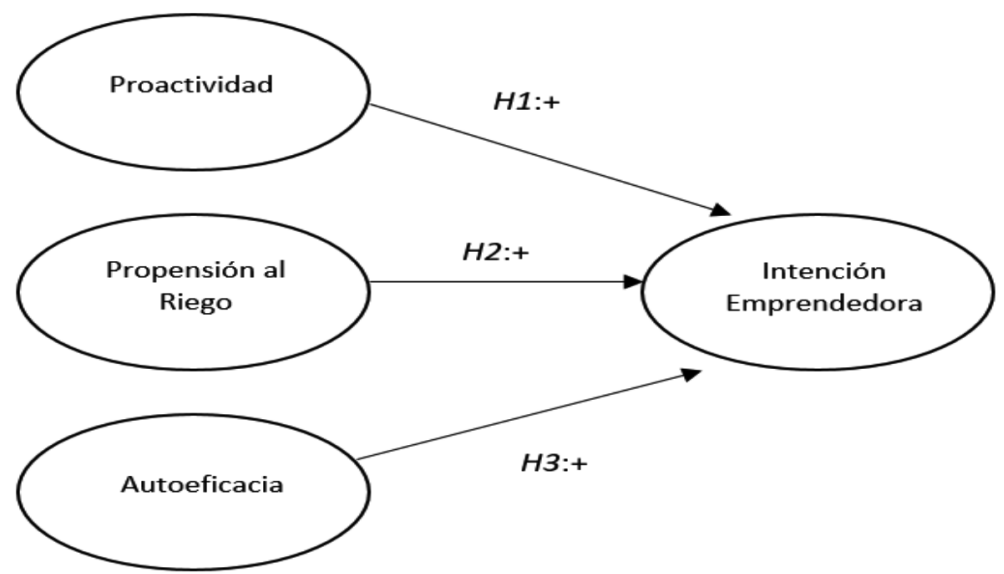

Figura 1. Modelo propuesto, basado en la Teoría de Comportamiento Planificado de Ajzen (1991).

A continuación, se detallan los aportes teóricos más importantes de las variables que fueron estudiadas en esta investigación.

Las intenciones emprendedoras exponen que la decisión de iniciar una actividad emprendedora requiere de una creencia preexistente de que dicha actividad es anhelada y realizable, unida con alguna propensión personal a actuar sobre las oportunidades y algún tipo de factor predominante. En el ámbito psicológico relacionado al fenómeno emprendedor, existen algunas variables enfocadas a la actitud conductual hacia el emprendimiento tal como la autoeficacia, 
la proactividad y la propensión al riesgo (Frese \& Gielnik, 2014; Scherer, Adams, Carley \& Wiebe, 1989; Prabhu, McGuire, Drost \& Kwong, 2012; Sánchez, 2011; Sánchez et al., 2005; Zhao, Seibert, \& Hills, 2005). Asimismo, existen otras variables que por su efecto influyen en el emprendimiento, por lo cual se han considerado para esta investigación como variables de control a los factores socio-demográficos como: (a) género, (b) edad, (c) estado civil, (d) nivel de ingresos y (e) campo de educación.

De acuerdo a Bateman \& Crant (1993) la personalidad proactiva se refiere a la tendencia a iniciar y mantener acciones que directamente alteran el contexto circundante. La acción implica crear un cambio, no simplemente anticiparlo. No se trata sólo de importantes atributos de flexibilidad y adaptabilidad hacia un futuro incierto. Ser proactivo es tomar la iniciativa en la mejora de los negocios. En el otro extremo, el comportamiento no proactivo incluye sentarse, dejar que otros hagan que las cosas sucedan y esperar pasivamente que el cambio impuesto externamente funciona bien (Bateman \& Crant, 1999). Por otro lado, Sánchez (2011) expresó que las personas proactivas identifican oportunidades y actúan sobre ellas, muestran iniciativa, toman acción directa hasta que hayan hecho un cambio significativo. Por el contrario, las personas no proactivas no identifican y actúan sobre las oportunidades de cambiar las cosas. Este término enfatiza la anticipación y prevención de los problemas antes de que ocurran y la orientación a la acción que incluye una interpretación creativa de las normas y un nivel de persistencia y paciencia para lograr el cambio (Sánchez, 2011; Sánchez et al., 2005).

Shapero y Sokol (1982) sugirieron que esta inclinación personal a actuar sobre las oportunidades es uno de los factores que pueden afectar la relación entre intención y el comportamiento al precipitar o facilitar la realización de las intenciones. En el contexto específico de la iniciativa empresarial, Crant (1996) encontró que las intenciones empresariales estaban positivamente asociadas con tener una personalidad proactiva.

Según Covin y Slevin (1989) el concepto de riesgo que toma la propensión al riesgo se ha relacionado con el espíritu empresarial. Es obvio que la actividad empresarial por definición implica algún tipo de riesgo. En este marco, la toma de riesgos se refiere a la voluntad del sujeto de comprometerse a fuentes de oportunidades con posibilidad de fracaso (Sánchez, 2011). La propensión al riesgo se ha relacionado con el individuo que desea ser emprendedor, por lo que se puede aseverar que toda actividad emprendedora tiene como condición primordial, la asunción de algún tipo de riesgo (Nieß \& Biemann, 2014).

Por otro lado, según Bandura (1986) las apreciaciones de autoeficacia parecen ser objetivas para entender la conducta planeada e intencional, dada su influencia en la formación de intenciones emprendedoras en los estudiantes universitarios. Este indicio hace que el análisis de 
la autoeficacia sea el más adecuado en las investigaciones sobre intenciones emprendedoras. Por otra parte, Ajzen (1991) argumentó que la autoeficacia tiene un lugar en los modelos de las intenciones de comportamiento previstas en general y en las intenciones del comportamiento planificado empresarial en particular, y es con frecuencia relacionada con el control del comportamiento percibido. De este modo, la percepción de autoeficacia parece ser crítico en la comprensión planificada y conducta intencional, dada su influencia en la formación de intenciones a través de la percepción de la situación de factibilidad (Sánchez et al., 2005; Zhao et al.2005). Esta premisa hace que el estudio de la autoeficacia sea particularmente investigado sobre las intenciones empresariales en los estudiantes universitarios. Asimismo, dado que la autoeficacia predice el reconocimiento de oportunidades, no es de extrañar que la percepción de autoeficacia aparezca como un elemento central en las intenciones emprendedoras (Prabhu et al., 2012; Scherer et al., 1989). De hecho, la investigación realizada en los últimos años ha demostrado con éxito el poder predictivo de la percepción de autoeficacia en la formación de las intenciones emprendedoras, debido a su influencia directa y su asociación con otras variables de interés en la explicación de las intenciones emprendedoras en los estudiantes universitarios (Prabhu et al., 2012; Sánchez, 2011; Sánchez et al., 2005; Zhao et al. 2005).

Se plantearon las siguientes hipótesis de investigación que permitieron comprobar los factores que inciden en las intenciones emprendedoras en los estudiantes universitarios de pregrado. Estas hipótesis se fundamentan en la TPB de Ajzen (1991):

H1: La proactividad incide positivamente en las intenciones emprendedoras de los estudiantes de pregrado.

H2: La propensión al riesgo incide positivamente en las intenciones emprendedoras de los estudiantes de pregrado.

H3: La autoeficacia incide positivamente en las intenciones emprendedoras de los estudiantes de pregrado.

\section{Método}

En esta investigación se estudió la influencia de la autoeficacia (AUTO), propensión al riesgo (RIESGO) y proactividad (PROACT) en la intención emprendedora (INTE_EMPREN) de los estudiantes universitarios. Se propuso en modelo basado en la TPB de Ajzen (1991, 2011), tomando como unidad de análisis una muestra no probabilístico voluntario a los estudiantes universitarios de las instituciones de educación superior de Ecuador. La muestra utilizada fue de 603 estudiantes de universidades públicas y privadas de las provincias de Guayas, Pichincha y Azuay. 
Se tomó como población a investigar a los estudiantes de ocho universidades de las provincias de Guayas, Pichincha y Azuay. Las universidades que formaron parte del levantamiento de los datos son: (a) Universidad Politécnica Salesiana-UPS, (b) Universidad Católica de Cuenca-UCACUE, (c) Escuela Superior Politécnica del Litoral-ESPOL, (d) Universidad Católica Santiago de Guayaquil-UCSG, (e) Universidad de Guayaquil-UG, (f) Universidad Central de Ecuador-UCE, (g) Escuela Politécnica Nacional-EPN y (h) Pontificia Universidad Católica del Ecuador-PUCE. El tipo de muestreo que se utilizó en esta investigación es no probabilístico voluntario, por medio de la técnica de auto-selección (Saunders, Lewis, \& Thornhill, 2012). Por medio de la aplicación de la encuesta, se obtuvo 603 encuestas válidas de estudiantes que participaron de manera voluntaria para la aplicación de la encuesta.

El instrumento en esta investigación se basó en un cuestionario de preguntas dirigidas a los integrantes de la muestra antes indicada. Para el levantamiento de datos se utilizó un instrumento de medición ajustado para el contexto de los estudiantes universitarios de Ecuador. Para asegurar la validez del instrumento de medición traducido, se realizó traducción inversa del español al inglés para confirmar el sentido original de las preguntas (Murray, Yong, \& Kotabe, 2011). Se realizó una prueba piloto para evaluar el entendimiento y la estructura de las preguntas de cada factor previo a la aplicación de la encuesta. A continuación, se especifica la utilización del cuadernillo por cada variable.

Intenciones emprendedoras: Mediante una escala tipo Likert de 1 a 5, con un ítem se mide a los estudiantes la posibilidad de crear su propio negocio.

Proactividad: De acuerdo a la escala de Personalidad Proactiva de Seibert et al. (1999, 2001) dividido en 10 ítems que mide la propensión individual hacia la conducta proactiva mediante una escala tipo Likert de 1 a 5 desde "completamente en desacuerdo" (1) a "completamente de acuerdo" (5).

Propensión al riesgo: De acuerdo a Rohrmann (1997) mediante el Cuestionario de Orientación al Riesgo (ROQ) dividido en 12 ítems que evalúan la tendencia general de los individuos a asumir riesgo mediante una escala tipo Likert de 1 a 5 desde "completamente en desacuerdo" (1) a "completamente de acuerdo" (5).

Autoeficacia: Mediante una escala tipo Likert de 1 a 5 desde "completamente incapaz" (1) a "completamente capaz" (5), de acuerdo a la escala Entrepreneurial Self-Efficacy (ESE) dividida por 23 ítems por parte de De Noble et al. (1999), que mide a los individuos en la creencia en sus propias habilidades para realizar las tareas demandadas para la creación de un negocio.

Para asegurar la confiabilidad del cuestionario incluido en el instrumento de investigación se analizó su consistencia interna por el coeficiente de alfa de Cronbach y del coeficiente de 
confiabilidad compuesta. Debido a que los factores PROACT y AUTO tienen más de 10 ítems cada uno, los coeficientes de alfa de Cronbach podrían estar subestimados. Por lo tanto, se hizo un análisis conjunto de confiabilidad por alfa de Cronbach y por confiabilidad compuesta (Hair, Hult, Ringle, \& Sarstedt, 2014).

Para asegurar la representatividad de las escalas en el contexto de los estudiantes universitarios de Ecuador se realizó un refinamiento de constructos. Para esto, se realizó un análisis factorial confirmatorio (AFC) para evaluar la contribución individual de las variables observables en la construcción de los factores (Lattin, Carroll, \& Green, 2003). La aplicación del AFC permitió reducir la dimensionalidad de los factores y ajustar las escalas para el estudio de los determinantes de la intención emprendedora de los estudiantes universitarios de Ecuador. Para ello, se propuso un modelo basado en la TPB de Ajzen (1991, 2011), por medio del cual se estudió la influencia de la autoeficacia (AUTO), la propensión al riesgo (RIESGO) y la proactividad (PROACT) en la intención emprendedora (INTE_MPREN) de los estudiantes universitarios. Para probar las hipótesis del modelo, se estimó un modelo de ecuaciones estructurales. Por medio de la estimación del modelo estructural, se buscó probar la influencia positiva y significativa de la autoeficacia, propensión al riesgo y proactividad en la intención emprendedora de estudiantes universitarios de Ecuador.

Previo a la estimación del modelo de medición por AFC y del modelo de ecuaciones estructurales fue necesario conocer el comportamiento de los datos para la elección del método de estimación (Shumacker \& Lomax, 2016). Para comprobar si los datos de las variables observables obtenidas de la encuesta se distribuían de manera normal, se realizó un análisis visual y la prueba de Kolmogorv- Smirnov (K-S). Por medio del análisis visual, se encontró que los datos de las variables observables tenían una distribución leptocúrtica con asimetría negativa, la cual constituyó una primera evidencia de distribución no normal de los datos (Ghasemi \& Zahediasl, 2012). Además, para realizar un análisis más confiable de normalidad univariada, se aplicó la prueba de K-S que es la mejor alternativa en situaciones en donde se tienen más de 30 registros (Long, Kara, \& Splillan, 2016). Por medio de la prueba de K-S se obtuvieron $p$-values $<.05$, con lo cual se rechazaron las hipótesis de distribución normal de las variables.

Ante la evidencia de la no normalidad de los datos de las variables observables, se identificaron los valores atípicos que pudieran afectar el comportamiento normal de los datos (Penny, 1996). Para la identificación de valores atípicos se utilizó la prueba de distancia de Malahanobis, tomando como valores atípicos los p-values <.001. Se identificó que el 13.9\% de los registros correspondieron a valores atípicos. Luego de identificar los valores atípicos, fueron eliminados de la base original, sin que esto mejore la distribución no normal de los datos. 
Para conocer la intensidad de la no normalidad de los datos, se evaluó la normalidad multivariada de los datos por medio la prueba de Mardia. Por medio del complemento Real Statistics para Excel se encontró la no normalidad multivariante severa por medio del análisis de la curtosis y asimetría multivariante (Zaiontz, 2017). Ante esta situación, Mínimo Parciales Cuadrados (PLS por sus siglas en inglés) es un método basado en varianzas que permite la estimación de modelos de ecuaciones estructurales en situaciones en donde los datos se distribuyen de manera no normal y se tienen valores atípicos de respuesta (Ringle, Sarstedt, \& Straub, 2012).

Para confirmar que los datos permitían realizar un análisis factorial, se analizó la adecuación muestral de Kaiser-Meyer-Olkin (KMO) y se realizó la prueba de esfericidad de Barttlet (Montoya, 2007). Se obtuvo un valor KMO de .857, con lo cual se superó el valor crítico recomendable de .8 . Por otro lado, se obtuvo un $p$-value $<.05$ en la prueba de Barttlet, lo cual sugiere que la matriz de correlaciones de las variables observables no corresponde a una matriz identidad. De esta forma, se justifica la adecuación de los datos para realizar un análisis factorial. Antes del realizar el AFC, se evaluó la confiabilidad de las escalas con la prueba alfa de Cronbach, encontrando valores por debajo del valor crítico de .7 para la confirmación de escalas probadas en investigaciones anteriores. Luego de este análisis se encontró que los factores de relaciones con inversionistas (F3), desafíos (F5) y desarrollo de recursos humanos (F6), dimensiones del factor de segundo orden autoeficacia, tuvieron consistencia interna por debajo de .7 (ver Tabla 1). Se obtuvo valores de fiabilidad compuesta aceptables por encima de 7. Sin embargo, por existir valores de varianza extraída media por debajo del valor crítico de .5 , fue necesario refinar los constructos por medio de la evaluación de las cargas factoriales.

Tabla 1

Análisis de Confiabilidad de Escalas - Constructos Originales

\begin{tabular}{|c|c|c|c|c|}
\hline & & $\begin{array}{l}\text { Alfa de } \\
\text { Cronbach }\end{array}$ & $\begin{array}{l}\text { Fiabilidad } \\
\text { compuesta }\end{array}$ & $\begin{array}{l}\text { Varianza } \\
\text { extraída } \\
\text { media (AVE) }\end{array}$ \\
\hline \multicolumn{2}{|c|}{ Autoeficacia (AUTO) } & 0.929 & 0.938 & 0.415 \\
\hline \multirow{6}{*}{ AUTO } & Desarrollo de nuevos productos (F1) & 0.865 & 0.897 & 0.558 \\
\hline & Entorno Innovador (F2) & 0.714 & 0.818 & 0.546 \\
\hline & Relaciones con Inversionistas (F3) & 0.627 & 0.798 & 0.585 \\
\hline & Definición de Objetivos (F4) & 0.758 & 0.861 & 0.674 \\
\hline & Desafíos (F5) & 0.510 & 0.733 & 0.498 \\
\hline & Desarrollo de Recursos Humanos (F6) & 0.412 & 0.719 & 0.487 \\
\hline \multicolumn{2}{|c|}{ Proactividad (PROACT) } & 0.849 & 0.810 & 0.327 \\
\hline \multicolumn{2}{|c|}{ Propensión al Riesgo (RIESGO) } & 0.735 & 0.761 & 0.283 \\
\hline
\end{tabular}


En la estimación del modelo de medición por medio del algoritmo PLS, se identificaron las variables observables que tienen una baja contribución en la construcción de los factores de proactividad, autoeficacia y propensión al riesgo. Se tomó .7 como valor crítico para las cargas factoriales, esto permitió refinar los constructos para el caso de los estudiantes universitarios de Ecuador. En el Apéndice A, se presentan las preguntas correspondientes a: (a) las variables observables A1 a la A23 correspondientes a la autoeficacia; (b) variables R1a R12 correspondiente a propensión al riesgo, de las cuales R2, R4, R6 y R12 se invirtió el sentido de las escalas para mantener la misma connotación de las preguntas y (c) variables PRO1 a PRO10 correspondiente a la proactividad.

Luego de eliminar las variables con bajas cargas factoriales, se obtuvieron escalas con una mejor consistencia interna y con mayor nivel de parsimonia. Como se muestra en la Tabla 2, entre las seis dimensiones de autoeficacia propuestas por De Noble et al. (1999), el factor desafíos (F5) tuvo una consistencia interna de .579 y el factor desarrollo de recursos humanos (F6) de .632. Pese a que .7 es nivel crítico de consistencia interna comúnmente aceptable, cuando se confirman las estructuras factoriales propuestas por la literatura, se acepta como válido aceptar como nivel crítico .6 cuando la investigación se realiza en entornos poco estudiados Kline (2011). En el análisis de validez discriminante, por medio del criterio de Fornell-Larker y de cargas cruzadas, se decidió eliminar el factor desafíos (F5) por tener una baja consistencia interna y porque sus ítems tienen altas cargas en otros factores de la Autoeficacia (Lloret, Ferreres-Travers, Hernández-Baeza, \& Tomá-Miguel, 2014).

Tabla 2

Análisis de Confiabilidad de Escalas - Modelo de Medición Reducido

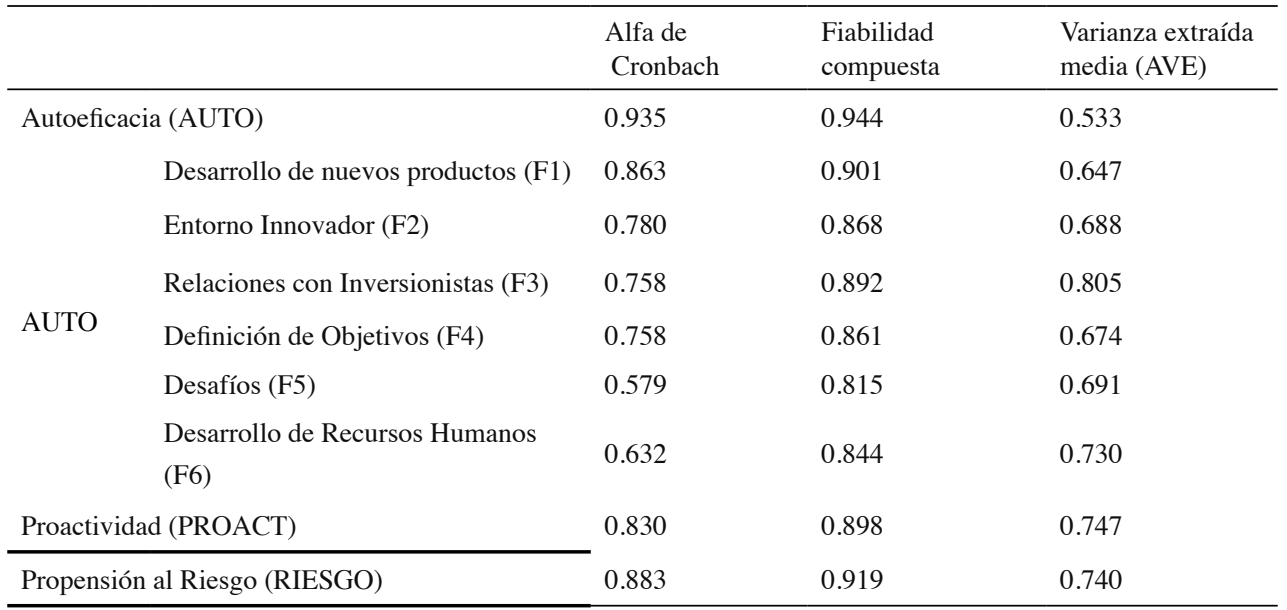


Para complementar el análisis de la confiabilidad de las escalas de los factores del modelo se realizó el análisis de validez discriminante de Fornell y Larker (1981), la validez convergente por medio de la varianza extraída media (AVE). En la Tabla 2 se muestra los valores de confiabilidad compuesta por encima de .815 y varianzas extraídas superior a .533 . Los valores AVE mayores a .5 evidencian las variables de los factores explican más de la mitad de la varianza del factor (Hair et al., 2014). En la Tabla 3 se presentan los resultados del análisis de validez discriminante según el criterio de Fornell y Larker (1981), en el cual se demuestra que la raíz cuadrada de las varianzas extraídas de variables latentes es mayor que su correlación con otros factores del modelo. De esta forma, se confirma que la confiabilidad y validez de las escalas que serán utilizadas en la estimación del modelo estructural.

Tabla 3

Validez Discriminante - Criterio Fornell y Larker (1981)

\begin{tabular}{|c|c|c|c|c|c|c|c|c|c|}
\hline & AUTO & $\mathrm{F} 1$ & $\mathrm{~F} 2$ & F3 & $\mathrm{F} 4$ & F6 & $\begin{array}{l}\text { INTEN } \\
\text { EMPREN }\end{array}$ & PROACT & RIESGO \\
\hline AUTO & 0.730 & & & & & & & & \\
\hline $\mathrm{F} 1$ & 0.946 & 0.804 & & & & & & & \\
\hline $\mathrm{F} 2$ & 0.767 & 0.723 & 0.829 & & & & & & \\
\hline F3 & 0.878 & 0.776 & 0.536 & 0.897 & & & & & \\
\hline F4 & 0.919 & 0.793 & 0.658 & 0.783 & 0.821 & & & & \\
\hline F6 & 0.829 & 0.725 & 0.405 & 0.769 & 0.786 & 0.854 & & & \\
\hline $\begin{array}{l}\text { INTEN_ } \\
\text { EMPREN }\end{array}$ & 0.642 & 0.529 & 0.378 & 0.731 & 0.605 & 0.621 & 1.000 & & \\
\hline PROACT & 0.772 & 0.684 & 0.537 & 0.785 & 0.722 & 0.663 & 0.763 & 0.864 & \\
\hline RIESGO & 0.740 & 0.635 & 0.512 & 0.679 & 0.767 & 0.654 & 0.569 & 0.707 & 0.860 \\
\hline
\end{tabular}

Nota: Datos correspondientes a la raíz cuadrada de la varianzas extraídas y correlaciones entre factores del modelo estructural.

Una vez obtenidas las escalas confiables y válidas, por medio del proceso de refinamiento por AFC, se estimó un modelo de ecuaciones estructurales PLS-SEM. PLS-SEM en un método para el análisis utilizado en sistemas de información gerencial, que ha sido ampliamente utilizado y aceptado por la comunidad científica desde el lanzamiento del paquete estadístico SmartPLS (Hair, Hult, Ringle, \& Sarstedt, 2014; Ringle, Sarstedt, \& Straub, 2012). 
En la Figura 2, se presentan los coeficientes de ruta obtenidos de la estimación del modelo de ecuaciones estructural por medio del algoritmo PLS. Lattin et al. (2003) sugiere aceptar coeficientes de ruta a los valores mayores a 20 . Sin embargo, ante la existencia de datos que tengan una distribución no normal multivariada, se puede llegar a cometer error de Tipo I por la generación de estimadores poco confiables (Brown, 2015). Sin embargo, en el caso de tener datos con distribución no normal multivariada, Ringle et al. (2012) y Kwong y Kay (2013) sugieren que el contraste de hipótesis de influencia, por medio de estimaciones de ecuaciones estructurales por PLS, se realice la estimación por bootstrapping con 5 mil sub-muestras para para el contraste de hipótesis por medio del análisis de $p$ valores generado por prueba $t$. El resultado de la estimación por bootstrapping con cinco mil sub-muestras se muestra en la Tabla 4.

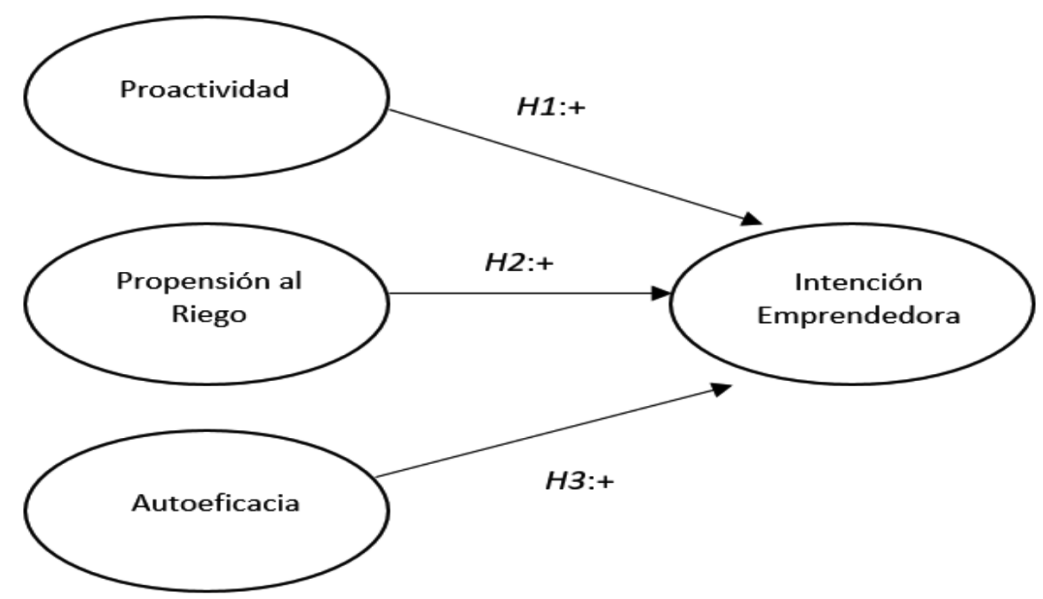

Figura 2. Resultados de estimación por Algoritmo PLS 
Tabla 4

Resultados Estimación por Bootstrapping

\begin{tabular}{llllll}
\hline & $\begin{array}{l}\text { Muestra } \\
\text { original (O) }\end{array}$ & $\begin{array}{l}\text { Media de la } \\
\text { muestra (M) }\end{array}$ & $\begin{array}{l}\text { Desviación } \\
\text { estándar } \\
\text { (STDEV) }\end{array}$ & $\begin{array}{l}\text { Estadísticos t } \\
\text { (I O/STDEVI) }\end{array}$ & P Valores \\
$\begin{array}{l}\text { H1: PROACT -> INT- } \\
\text { EN_EMPREN }\end{array}$ & 0.657 & 0.659 & 0.053 & 12.361 & $.000^{* *}$ \\
$\begin{array}{l}\text { H2: RIESGO -> INT- } \\
\text { EN_EMPREN }\end{array}$ & 0.011 & 0.013 & 0.046 & 0.232 & $.816 \mathrm{NS}$ \\
$\begin{array}{l}\text { H3: AUTO -> INT- } \\
\text { EN_EMPREN }\end{array}$ & 0.127 & 0.122 & 0.063 & 2.023 & $.043 *$ \\
AUTO -> F1 & 0.946 & 0.946 & 0.003 & 301.372 & $.000^{* *}$ \\
AUTO -> F2 & 0.767 & 0.768 & 0.015 & 50.225 & $.000 * *$ \\
AUTO -> F3 & 0.878 & 0.878 & 0.013 & 65.451 & $.000 * *$ \\
AUTO -> F4 & 0.919 & 0.918 & 0.009 & 104.801 & $.000^{* *}$ \\
AUTO -> F6 & 0.829 & 0.829 & 0.018 & 45.101 & $.000 * *$ \\
\hline
\end{tabular}

Nota: ** significativo al $.01 ; *$ significativo al .05 ; NS no significativo.

Por medio de la estimación del modelo por algoritmo PLS y por bootstrapping, se pudo comprobar las hipótesis 1 y la hipótesis 3 del presente trabajo. Es decir, que proactividad () como factor reflectivo unidimensional y que la autoeficacia () como factor reflectivo de segundo orden, tienen una influencia positiva y significativa en la intención emprendedora de los estudiantes universitarios de Ecuador. Por otro lado, con un coeficiente de .011 y un nivel de significancia superior al valor crítico de .05 , se rechazó la hipótesis () de influencia positiva y significativa de la propensión al riesgo en la intención emprendedora de los estudiantes universitarios de Ecuador.

Para evaluar la calidad de la estimación del modelo estructural se evaluó el coeficiente de determinación, el cual tuvo un valor de .589. Pese a que no existe un valor crítico de, se considera que el nivel de predictibilidad del modelo es muy bueno. Aunque el objetivo de la estimación por PLS es maximizar el , la calidad del modelo también debe ser evaluado por medio del efecto tamaño que mide la variación del coeficiente de determinación ante la eliminación de un factor exógeno (Ringle et al., 2012). El valor del factor proactividad (PROACT) fue .382 lo cual representa una alta capacidad predictiva en la intención emprendedora de los estudiantes universitarios de Ecuador. Por otro lado, el factor autoeficacia (AUTO) pese a tener una influencia significativa en la intención emprendedora, su capacidad predictiva es baja con un valor de .013 . 
Además, se realizó el análisis de inflación de la varianza (VIF) para evaluar la colinealidad de las variables del modelo de medición y los valores VIF para el modelo estructural (Garson, 2016). Se tomó un valor crítico de VIF de cinco, encontrando no tener problema de colinealidad en el modelo estructural, pero encontrando un valor VIF de 5.092 para la variable observable A3. Ante la evidencia de colinealidad, se sugiere agrupar o eliminar variables observables (Garson, 2016). Sin embargo, se considera que, por estar tan cercano al valor crítico de cinco, se mantuvo en el factor Desarrollo de Nuevos Productos (F1), ya que luego de estimar el modelo sin la variable correspondiente a la capacidad para reconocer nuevas oportunidades (A3) no mejoró la calidad predictiva del modelo.

Pese a que el modelo tiene una importante capacidad predictiva en la intención emprendedora de estudiantes universitarios de pregrado de Ecuador, para profundizar en la intensidad de las relaciones se realizó un análisis de heterogeneidad del modelo. Para esto, se realizó un análisis de moderación incluyendo variables de características de la unidad de análisis. Se utilizó a la edad y genero para realizar el análisis de moderación por medio del paquete estadístico SmartPLS versión 3. Por medio del análisis de moderación, se encontró que la intensidad en la relación entre la percepción de autoeficacia y la intención emprendedora no se ve moderada por la edad ni por el género. Además, se encontró que la intensidad de la relación entre proactividad y la intención emprendedora no es diferente para hombres y mujeres ni para los estudiantes universitarios de distintas edades.

\section{Discusión}

En este trabajo se estudió la influencia del comportamiento planificado en la intención emprendedora de estudiantes universitarios de pregrado. Para ello, se estudiaron las dimensiones del comportamiento planificado propuesto por Ajzen $(1991,2011)$, considerando que la cultura del Ecuador se caracteriza por contar con distintos hábitos y a pesar que esta teoría ha sido utilizada para predecir el comportamiento de los individuos, dichos estudios se han centrado en economías desarrolladas utilizando como población de análisis a individuos que cuentan con formación universitaria (Ambad \& Damit, 2016; Goethner et al., 2012; Kakouris, 2016; Shirokova et al., 2015). La TPB ha sido utilizada en distintos contextos, por lo que se ha sugerido que se evalúe su consistencia externa en contextos poco estudiados.

Por medio del análisis factorial confirmatorio se pudo validar las escalas para el contexto de los estudiantes universitarios de pregrado de Ecuador. Para profundizar en los resultados, se operacionalizó al factor autoeficacia como constructo de segundo orden construido por 
las seis dimensiones de autoeficacia propuestas por De Noble et al. (1999): (a) desarrollo de nuevos productos, (b) entorno innovador, (c) relaciones con inversionistas, (d) definición de objetivos, (e) desafíos y (f) desarrollo de recursos humanos. Por medio de este análisis, se encontró que, para los estudiantes universitarios de Ecuador, la autoeficacia no implica el planteamiento de desafíos.

Por otro lado, en el presente estudio se pudo demostrar que la intención emprendedora puede ser explicada por el comportamiento de autoeficacia y la proactividad de los estudiantes universitarios de Ecuador. Las dimensiones del comportamiento planificado de Ajzen explican el .589 de variación de los resultados de la intención emprendedora para el caso de los estudiantes universitarios de Ecuador, según el análisis el coeficiente de determinación . En la literatura que se encuentra en la actualidad (Brooke, Mohd, \& Abu, 2017; Bullough, Renko \& Myatt, 2014; Kaczmarek \& Kaczmarek-Kurczak, 2016; Prabhu et al., 2012; Shinnar et al., 2014) se utilizan otras variables que tienen mayor incidencia en las intenciones emprendedoras en los estudiantes universitarios, como la autoeficacia y la proactividad que tienen cabida en los modelos de intenciones del comportamiento planificado.

De acuerdo a la Figura 2, la H1 y H3 pudieron ser comprobadas para el caso de los estudiantes universitarios de Ecuador y son consistentes con los estudios previos realizados (Drnovšek, Wincent, \& Cardon, 2010; Frese \& Gielnik, 2014; Prabhu et al., 2012; Sánchez, 2011; Sánchez et al., 2005; Zhao et al., 2005), con lo cual precisa la pertinencia de la intención emprendedora en la investigación. Por otro lado, la $\mathrm{H} 2$ no pudo ser comprobada para el caso de estudiantes universitarios de Ecuador, incluso se realizó un análisis de heterogeneidad del modelo para determinar si la relación podría ser confirmada en estudiantes según su género y edad; lo que correspondería a un resultado novedoso ya que no es consistente con los estudios previos (Busenitz, 1999; Larson, Bussom, Vicars, \& Jauch, 1986; Nieß \& Biemann, 2014; Sánchez, 2011; Soria-Barreto et al., 2016). Los resultados de la estimación heterogeneidad del modelo por sexo y por edad, pueden deberse a las características de las muestra correspondiente a estudiantes de ocho universidades distintas y de distintas carreras.

Por lo tanto, el aporte científico a la academia sería que la TPB logra explicar la intención emprendedora en estudiantes universitarios por intermedio de dos de sus tres dimensiones.

Otra de los aportes en esta investigación es la inclusión del género como variable moderadora en la intensidad de las relaciones de las dimensiones del comportamiento planificado y la intención emprendedora. Esto fue posible por la utilización de PLS como método de estimación, el cual permite la inclusión de variables binarias en el análisis de heterogeneidad del modelo (Hair et al., 2014). 


\section{Conclusiones}

En esta investigación se estudió la intención emprendedora de los estudiantes universitarios de pregrado de Ecuador, usando la TPB de Ajzen $(1991,2011)$. Para el estudio de la intención emprendedora se ha analizado las creencias conductuales, normativas y de control. Para el desarrollo de la investigación, se plantearon tres hipótesis las cuales se detallan a continuación.

Se acepta la hipótesis que la proactividad tiene una influencia positiva y significativa en el la intención emprendedora de estudiantes universitarios de pregrado en Ecuador. Por medio del refinamiento de constructos por AFC, se encontró que, para los estudiantes universitarios de Ecuador, la proactividad implica el comportamiento dirigido hacia la solución de problemas a partir de ideas y acciones que incluso vayan en contra de lo establecido. Con ello, los estudiantes con mayor intención emprendedora muestran tener confianza en sí mismos con la capacidad de defender sus ideas que consideren les llevará a lograr sus objetivos.

Se rechaza la hipótesis que la propensión al riesgo tiene una influencia positiva y significativa en el la intención emprendedora de estudiantes universitarios de pregrado en Ecuador. Para el caso de los estudiantes de pregrado en Ecuador, la propensión al riesgo, medida con la escala inversa del constructo original, implica no estar dispuesto a asumir riegos no calculados y a la tendencia a evaluar los escenarios más desfavorables de sus acciones. Por ello, el comportamiento adverso a asumir riesgos no influencia la intención de emprender para el caso de estudiantes de pregrado de universidades ecuatorianas.

Para el caso de estudiantes universitarios de pregrado de Ecuador, se acepta la hipótesis de que la autoeficacia tiene una influencia positiva y significativa en la intención emprendedora. En este estudio, la autoeficacia es un constructo reflectivo de segundo orden construido por las dimensiones de desarrollo de nuevos productos, entorno innovador, relaciones con los inversionistas, definición de objetivos y desarrollo de recursos humanos.

Los resultados de la investigación contribuyen al estudio de la intención emprendedora desde la perspectiva de la TPB, ya que ha sido poco estudiado en el contexto de los estudiantes universitarios en economías no desarrolladas. Además, los resultados obtenidos tienen una implicancia práctica porque permite conocer el complimiento de la misión de las universidades ecuatorianas respecto a la formación de emprendedores.

De los resultados obtenidos, la autoeficacia es la convicción de cada individuo para organizar y efectuar acciones para el complimiento de objetivos desafiantes relacionados al desarrollo de nuevos productos, innovación, desarrollo de recursos humanos y al fomento de relaciones interpersonales. La autoeficacia en esta investigación es medida como la creencia de los estudiantes sobre sus propias habilidades para la creación de un negocio. Para las au- 
toridades universitarias resulta interesante conocer que, para los estudiantes, la autoeficacia no implica autopercepción de habilidades para el trabajo en equipo, la adaptación ante los cambios de entorno y de resiliencia, es decir que se deben enfocar al cumplimiento de los objetivos empresariales.

Para los estudiantes universitarios de Ecuador, la proactividad implica la propensión individual hacia la resolución de problemas, defensa de ideas y tenacidad para cumplir con los objetivos. Por otro lado, los estudiantes universitarios que evidencian tener una actitud conservadora hacia la propensión al riesgo, por medio de la toma de decisiones seguras con riesgos calculados, no tienen la intención de emprender, es decir que se enfocan a conseguir un empleo bajo dependencia. Según los resultados obtenidos, la proactividad es el factor que más contribuye a la intención de crear un negocio en un corto plazo. Por otro lado, la intención de crear un nuevo negocio también se ve influenciada de manera positiva y significativa por el autopercepción de habilidades necesarias para la creación de unos nuevos negocios. Sin embargo, esta percepción de habilidades no es un gran predictor de la intención emprendedora. Por último, la intención de emprender en un futuro cercano, no depende de la actitud hacia los riesgos relacionados a la toma de decisiones y acciones para la creación de un nuevo negocio.

En conclusión, la intención de emprender por parte de estudiantes universitarios depende principalmente de la proactividad hacia la resolución de problemas y a la persistencia para el logro de objetivos para el desarrollo de una idea de negocios. Por medio del análisis de moderación, se comprobó que la importancia de la proactividad y la autoeficacia para el emprendimiento no tiene una diferencia significativa en la intención emprendedora para estudiantes universitarios tanto hombres como mujeres de cualquier edad.

Una de las principales limitaciones de este estudio corresponde al tipo de muestreo utilizado para la aplicación de la encuesta. Los datos fueron levantados por medio de la aplicación de encuestas con medidas de percepción a estudiantes de distintas carreras y tipos de universidades, considerando que la muestra mantenga la misma composición de la población. Por otro lado, respecto a la temporalidad, los datos fueron levantados en único momento en el tiempo, por lo que no se logra relaciones de causalidad entre variables. Una de las limitaciones de los estudios de corte transversal se da en que las variables de comportamiento planificado y de intención emprendedora son levantadas al mismo tiempo.

Para la extensión del conocimiento de la intención emprendedora, se sugiere comparar los resultados de la presente investigación con estudiantes de maestría y doctorado para evaluar si existe una diferencia significativa en las dimensiones del comportamiento planificado y en su influencia en la intención emprendedora. Además, se sugiere realizar un estudio longitudinal para determinar si la formación académica que reciben los estudiantes influencia la relación 
del comportamiento planificado y la intención emprendedora. Asimismo, para extender el conocimiento de la intención emprendedora, se sugiere estudiar el efecto del tipo de carreras en la relación entre comportamiento planificado y la intención emprendedora. Por último, se sugiere analizar la intención emprendedora de los estudiantes universitarios por medio de variables personales como estilos de liderazgo y rasgos de personalidad utilizando el diseño no experimental con corte longitudinal.

\section{Referencias}

Ajzen, I. (1991). The theory of planned behavior. Organizational Behavior and Human Decision Processes, 50(2), $179-211$.

Ajzen, I. (2011). The theory of planned behaviour: reactions and reflections. Psychology \& Health, 26(9), 11131127. https://doi.org/10.1080/08870446.2011.613995

Ajzen, I., \& Fishbein, M. (1980). Understanding attitudes and predicting social behavior.

Al Mamun, A., Binti Che Nawi, N., Dewiendren, A., \& Fazira Binti, S. (2016). Examining the effects of entrepreneurial competencies on students' entrepreneurial intention. Mediterranean Journal of Social Sciences, 119127. https://doi.org/10.5901/mjss.2016.v7n2p119

Ambad, S., \& Damit, D. (2016). Determinants of entrepreneurial intention among undergraduate students in Malaysia. Procedia Economics and Finance, 37, 108-114. https://doi.org/10.1016/S2212-5671(16)30100-9

Arasteh, H., Enayati, T., Zameni, F., \& Khademloo, A. (2012). Entrepreneurial personality characteristics of university students: a case study. Procedia - Social and Behavioral Sciences, 46(1), 5736-5740. https://doi. org/10.1016/j.sbspro.2012.06.507

Bandura, A. (1986). The Social Foundations of Thought and Action. Englewood Cliffs, NJ: Prentice Hall.

Bandura, A. (1997). Self-efficacy: The Exercise of Control. New York: W. H. Freeman \& Co.

Bateman, S., \& Crant, J. (1993). The proactive component of organizational behavior. Journal of Organizational Behavior, 14(2), 103-118.

Bateman, S., \& Crant, J. (1999). Proactive behavior: meanings, impact, and recommendations. Business Horizons, 42(3), 63-70.

Bird, B. (1988). Implementing entrepreneurial ideas: the case for intention. Academy of Management Review, 13(3), 442-445.

Brooke, J., Mohd, R., \& Abu, B. (2017). Modelling knowledge sharing behaviour using self-efficacy as a mediator. European Journal of Training and Development, 41(2), 144-159. https://doi.org/10.1108/EJTD-04-2016-0021

Brown, T. (2015). Confirmatory Factor Analysis for Applied Research. (Second ed.). New York: The Guilford Press.

Bullough, A., Renko, M., \& Myatt, T. (2014). Danger zone entrepreneurs: the importance of resilience and self-efficacy for entrepreneurial intentions. Entrepreneurship Theory and Practice, 38(3), 473-499. https://doi. org/10.1111/etap.12006

Busenitz, L. (1999). Entrepreneurial risk and strategic decision making: it's a matter of perspective. Journal of Applied Behavioural Science, 35(3), 325-340.

Covin, J., \& Slevin, D. (1989). Strategic management of small firms in hostile and benign environments. Strategic Management Journal, 10(1), 75-87.

Crant, J. (1996). The proactive personality scale as a predictor of entrepreneurial intentions. Journal of Small Business Management, 34(3), 42-49. 
De Noble, A., Jung, J., \& Ehrlich, S. (1999). Entrepreneurial selfefficacy: the development of a measure and its relationship to entrepreneurial action. Frontiers of Entrepreneurship Research. Wellesley MA: Babson College.

Diez, S. (2016). La actitud conductual en las intenciones emprendedoras. Revista Empresarial, ICE-FEE-UCSG, 10(2), 42-48.

Drnovšek, M., Wincent, J., \& Cardon, M. (2010). Entrepreneurial self-efficacy and business start-up: developing a multi-dimensional definition. International Journal of Entrepreneurial Behavior \& Research, 16(4), 329-348. https://doi.org/10.1108/13552551011054516

Fayolle, A., \& Liñán, F. (2014). The future of research on entrepreneurial intentions. Journal of Business Research, 67(5), 663-666. https://doi.org/10.1016/j.jbusres.2013.11.024

Ferreira, J., Raposo, M., Gouveia, R., Dinis, A., \& do Paço, A. (2012). A model of entrepreneurial intention: an application of the psychological and behavioral approaches. Journal of Small Business and Enterprise Development, 19(3), 424-440. https://doi.org/10.1108/14626001211250144

Fishbein, M., \& Ajzen, I. (1975). Belief, attitude, intention and behavoir: An introduction to theory and research. Reading, MA: Addison-Wesley.

Frese, M., \& Gielnik, M. (2014). The psychology of entrepreneurship. Annual Review of Organizational Psychology and Organizational Behavior, 1(1), 413-438. http://doi.org/10.1146/annurev-orgpsych-031413-091326.

Garson, D. (2016). Partial least squares: Regression \& structural equation models. Asheboro: Statistical Associates Blue Book Series.

Ghasemi, A., \& Zahediasl, S. (2012). Normality Tests for Statistical Analysis: A Guide for Non-Statisticians. International Journal of Endocrinol Metabolism, 10(2), 486-489.

Hair, J., Hult, T., Ringle, C., \& Sarstedt, M. (2014). A premier on partial least squares structural equation modeling (PLS-SEM). Los Angeles: SAGE.

Hussain, A., \& Hashim, N. (2015). The impact of entrepreneurial alertness on entrepreneurial intentions. Journal of International Business Research and Marketing, 1(6), 7-11. https://doi.org/10.18775/jibrm.1849-8558.2015.16.3001

Israr, A., \& Hashim, N. (2015). Research on entrepreneurial intentions: an academic literature review and classification. Paradigms, 9(1), 9-28.

Kaczmarek, M., \& Kaczmarek-Kurczak, P. (2016). Personality traits and self-efficacy as predictors of business performance: a longitudinal study. Annals of Psychology, 19(1), 121-137. https://doi.org/10.18290/ rpsych.2016.19.1-4en

Kakouris, A. (2016). Exploring entrepreneurial conceptions, beliefs and intentions of Greek graduates. International Journal of Entrepreneurial Behavior \& Research, 22(1), 109-132. https://doi.org/10.1108/IJEBR-07-2014-0137

Kline, R. (2011). Principles and practice of structural equation modeling (Tercera ed.). New York: The Guilford Press.Krueger, N., \& Brazeal, D. (1994). Entrepreneurial potential and potential entrepreneurs. Entrepreneurship Theory and Practice, 18(1), 91-104

Krueger, N., Reilly, M., \& Carsrud, A. (2000). Competing models of entrepreneurial intentions. Journal of Business Venturing, 15(5), 411-432.

Kurato, D. (2008). Entrepreneurship, theory, process, and practice (8th ed.) Cengage Learning.

Küttim, M., Kallaste, M., Venesaar, U., \& Kiis, A. (2014). Entrepreneurship education at university level and students' entrepreneurial intentions. Procedia - Social and Behavioral Sciences, 110(5), 658-668. https://doi. org/10.1016/j.sbspro.2013.12.910

Kwong, K., \& Kay, W. (2013). Partial least squares structural equation modeling (PLS-SEM) techniques using SmartPLS. Marketing Bulletin, 24(1), 1-32.

Lanero, A., Vázquez, J., \& Muñoz-Adánez, A. (2015). A social cognitive model of entrepreneurial intentions in university students. Annals of Psychology, 31(1), 243-259. http://doi.org/10.6018/analesps.31.1.161461

Larson, L., Bussom, R., Vicars, W., \& Jauch, L. (1986). Proactive versus reactive manager: is the dichotomy realistic?. Journal of Management Studies, 23(4), 385-400. 
Lattin, J., Carroll, D., \& Green, P. (2003). Analyzing multivariate data. Canada: Cengage Learning.

Liñán, F., \& Fayolle, A. (2015). A systematic literature review on entrepreneurial intentions: citation, thematic analyses, and research agenda. International Entrepreneurship and Management Journal, 11(4), 907-933. https:// doi.org/10.1007/s11365-015-0356-5

Lloret, S., Ferreres-Travers, D., Hernández-Baeza, A., \& Tomá-Miguel, I. (2014). El análisis factorial exploratorio de los ítems: una guía práctica, revisada y actualizada. Anales de Psicología, 30(3), 1151-1169.

Long, Z., Kara, A., \& Splillan, J. (2016). Market orientation and firm performance: An empirical analysis of chinese IT enterprises. Sixth Asia-Pacific Conference on Global Business, Economics, Finance and Social Sciences (AP16Thai Conference), (págs. 1-18). Bangkok-Thailand.

Montoya, O. (2007). Application of the factorial analysis on the investigation of Mmarkets. Case of study. Scientia et Technica, 13(35), 281-286.

Murray, J., Yong, G., \& Kotabe, M. (2011). Market orientation and performance of export ventures: The process through marketing capabilities and competitive. Journal of the Academy of Marketing Science, 39(2), 252-269. doi: 10.1007/s11747-010-0195-4

Mustafa, M., Hernandez, E., Mahon, C., \& Chee, L. (2016). Entrepreneurial intentions of university students in an emerging economy: the influence of university support and proactive personality on students' entrepreneurial intention. Journal of Entrepreneurship in Emerging Economies, 8(2), 162-179. https://doi.org/10.1108/JEEE10-2015-0058

Nerlich, M. (1987). Ideology of adventure: studies in modern consciousness. Minneapolis: University of Minnesota Press.

Nieß, C., \& Biemann, T. (2014). The role of risk propensity in predicting self-employment. Journal of Applied Psychology, 99(5), 1000-1009. http://doi.org/10.1037/a0035992.

Penny, K. (1996). Appropriate critical values when testing for a single multivariate outliers by using the Mahalanobis distance. Applied Statistics, 45(1), 73-81.

Prabhu, V., McGuire, S. J., Drost, E., \& Kwong, K. (2012). Proactive personality and entrepreneurial intent: is entrepreneurial self-efficacy a mediator or moderator?. International Journal of Entrepreneurial Behavior \& Research, 18(5), 559-586. https://doi.org/10.1108/13552551211253937

Ringle, C., Sarstedt, M., \& Straub, D. (2012). A critical look at the use of PLS-SEM in MIS Quarterly. MIS Quarterly, 36(1), 1-19.

Rohrmann, B. (1997). Risk Orientation Questionnaire: Attitudes towards risk decisions (pre-test version). University of Melbourne.

Sánchez, J. (2011). University training for entrepreneurial competencies: its impact on intention of venture creation. International Entrepreneurship and Management Journal, 7(2), 239-254. https://doi.org/10.1007/s11365010-0156-X

Sánchez, J., Lanero, A., \& Yurrebaso, A. (2005). Determinant variables of the entrepreneurial intention in the university context. Revista de Psicología Social Aplicada, 15(1), 37-60.

Saunders, M., Lewis, P., \& Thornhill, A. (2012). Research methods for business students. Pearson.

Scherer, R., Adams, J., Carley, S., \& Wiebe, F. (1989). Role model performance effects on development of entrepreneurial career preference. Entrepreneurship Theory and Practice, 13(3), 53-81.

Schlaegel, C., \& Koenig, M. (2012). Determinants of entrepreneurial intent: a meta-analytic test and integration of competing models. Paper presented at the Academy of Management Annual Meeting, Boston, MA.

Schlaegel, C., \& Koenig, M. (2014). Determinants of entrepreneurial intent: a meta-analytic test and integration of competing models. Entrepreneurship Theory and Practice, 38(2), 291-332. https://doi.org/10.1111/etap.12087

Seibert, S., Crant, J., \& Kraimer, M. (1999). Proactive personality and career success. Journal of Applied Psychology, 84(3), 416-427.

Shapero, A., \& Sokol, L. (1982). The social dimensions of entrepreneurship. En C. Kent, D. Sexton \& K. H. Vesper (Eds.), Encyclopaedia of Entrepreneurship (pp. 72-90), Englewood Cliffs, NJ: Prentice Hall. 
Shinnar, R., Hsu, D., \& Powell, B. (2014). Self-efficacy, entrepreneurial intentions, and gender: assessing the impact of entrepreneurship education longitudinally. The International Journal of Management Education, 12(3), 561-570. https://doi.org/10.1016/j.ijme.2014.09.005

Shirokova, G., Osiyevskyy, O., \& Bogatyreva, K. (2015). Exploring the intention-behavior link in student entrepreneurship: moderating effects of individual and environmental characteristics. European Management Journal, 34(4), 386-399. .https://doi.org/10.1016/j.emj.2015.12.007

Shumacker, R., \& Lomax, R. (2016). A beginner's guide to structural equation modeling (Cuarta ed.). New York: Taylor \& Francis.

Stewart, A. (1990). The bigman metaphor for entrepreneurship: A "library tale" with morals on alternatives for further research. Organization Science, 1(2), 143-159.

Soria-Barreto, K., Zuniga-Jara, S., \& Ruiz-Campo, S. (2016). Entrepreneurial education and intention in university students: a case of study. Formación Universitaria, 9(1), 25-34. http://doi.org/10.4067/S071850062016000100004.

Van Gelderen, M., Brand, M., van Praag, M., Bodewes, W., Poutsma, E., \& van Gils, A. (2008). Explaining entrepreneurial intentions by means of the theory of planned behaviour. Career Development International, 13(6), 538-559.

Verheul, I., Block, J., Burmeister-Lamp, K., Thurik, R., Tiemeier, H., \& Turturea, R. (2015). ADHD-like behavior and entrepreneurial intentions. Small Business Economics, 45(1), 85-101. http://doi.org/10.1007/s11187-015$9642-4$.

Zaiontz, C. (10 de Junio de 2017). Real Statistics using Excel. Obtenido de http://www.real-statistics.com

Zhang, P., Wang, D., \& Owen, C. (2015). A study of entrepreneurial intention of university students. Entrepreneurship Research Journal, 5(1), 61-82. http://doi.org/10.1515/erj-2014-0004.

Zhao, H., Seibert, S., \& Hills, G. (2005). The mediating role of self-efficacy development of entrepreneurial intentions. Journal of Applied Psychology, 90(6), 1265-1272.

\section{Anexo}

\section{Cuestionario Propuesto}

PROACTIVAD

Mide la propensión individual hacia la conducta proactiva

\begin{tabular}{lccccc}
\hline \multirow{2}{*}{ ESCALA } & $\begin{array}{c}\text { Completamente } \\
\text { en desacuerdo }\end{array}$ & $\begin{array}{c}\text { En } \\
\text { desacuerdo }\end{array}$ & $\begin{array}{c}\text { Ni de acuerdo } \\
\text { ni en } \\
\text { desacuerdo }\end{array}$ & De acuerdo & $\begin{array}{c}\text { Completamente } \\
\text { de acuerdo }\end{array}$ \\
\cline { 2 - 6 } & 1 & 2 & 3 & 4 & 5 \\
\hline
\end{tabular}

Estoy constantemente en la búsqueda de nuevas formas de mejorar mi vida.

Donde he estado laborando, he sido parte de una fuerza para el cambio constructivo.

No hay nada más emocionante que ver que mis ideas se conviertan en realidad. 
Si veo algo que no me gusta, lo arreglo.

No importa cuáles son las probabilidades; si creo en algo, haré que suceda.

Me encanta defender mis ideas, incluso en contra de la oposición de los demás.

Me destaco en la identificación de oportunidades.

Siempre estoy en busca de mejores formas de hacer las cosas.

Si creo en una idea, ningún obstáculo me impedirá hacer que esta suceda.

Puedo detectar una buena oportunidad antes que otros lo hagan.

\section{PROPENSION AL RIESGO}

Se evaluará la tendencia general de los individuos a asumir riesgo

Tengo bastante cuidado cuando hago planes y cuando actúo en ellos.

Sigo el lema, "nada arriesga, nada gana".

No tengo mucha simpatía por las decisiones de aventura.

Si una tarea parece interesante, yo podría elegir hacerla, incluso si no estoy seguro que puedo manejarla.

No me gusta poner algo en juego, yo preferiría estar del lado seguro.

Aun cuando sé que mis posibilidades son limitadas, probaría mi suerte.

En mi trabajo sólo pongo metas pequeñas para que pueda alcanzarlos sin dificultad.

Expreso mi opinión, aunque la mayoría de las personas tengas puntos de vista opuestos. 
Mis decisiones se realizan siempre con cuidado y precisión.

Me gustaría actuar con mi jefe en el trabajo un poco más de tiempo a fin de demostrar mi competencia, a pesar del riesgo de cometer errores.

Tendencia a imaginar los resultados desfavorables de mis acciones El éxito me hace tomar mayores riesgos

AUTOEFICACIA

Mide a los individuos en la creencia de sus propias habilidades para realizar las tareas demandadas para la creación de un negocio

Trabajar eficazmente bajo presión

Relaciones favorables

Reconocer nuevas oportunidades

Empleado clave

Visión y valores organizacionales

Mejorar los productos existentes

Relaciones con personas clave

Áreas de crecimiento personal

Planificación del personal

Inspirar a otros

Tolerar los cambios inesperados

Resolver problemas corrientes

Identificar recursos potenciales

Que las personas sean su propio jefe

Persistir frente a la adversidad

Satisfacer las necesidades no cubiertas de los clientes

Acciones rápidas para perseguir oportunidades

Que las personas intenten hacer cosas 
Usar antiguos conceptos de nueva forma

Determinar si el negocio va bien

Alentar a las personas para que tomen decisiones

Identificar y construir equipos de gestión

Formar asociaciones o alianzas

\section{INTENCION EMPRENDEDORA}

Tiene la intención de crear su pro-

pio negocio en un plazo de cuatro años. 